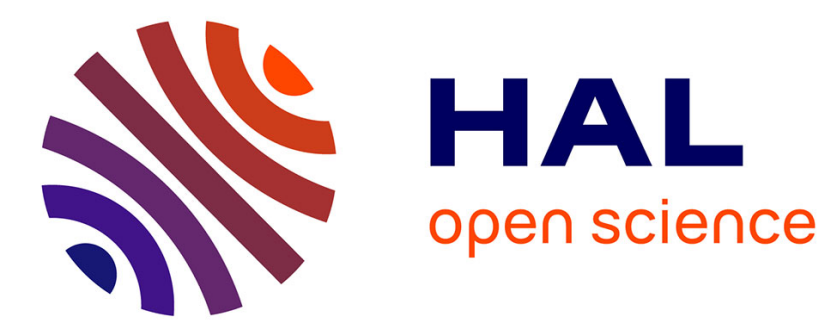

\title{
Un réseau de fossés défensifs aux origines de Lyon
} Bernard Mandy, Martine Genin, Catherine Godard, Sophie Krausz, Gérard Sandoz, Philippe Thirion

\section{To cite this version:}

Bernard Mandy, Martine Genin, Catherine Godard, Sophie Krausz, Gérard Sandoz, et al.. Un réseau de fossés défensifs aux origines de Lyon. Gallia - Fouilles et monuments archéologiques en France métropolitaine, 1988, 45, pp.49-66. 10.3406/galia.1987.2879 . hal-01772896

\section{HAL Id: hal-01772896 \\ https://hal.science/hal-01772896}

Submitted on 14 Jan 2020

HAL is a multi-disciplinary open access archive for the deposit and dissemination of scientific research documents, whether they are published or not. The documents may come from teaching and research institutions in France or abroad, or from public or private research centers.
L'archive ouverte pluridisciplinaire HAL, est destinée au dépôt et à la diffusion de documents scientifiques de niveau recherche, publiés ou non, émanant des établissements d'enseignement et de recherche français ou étrangers, des laboratoires publics ou privés.

\section{(ㅇ)(1) $\$$}

Distributed under a Creative Commons Attribution - NonCommercial - NoDerivatives| 4.0 


\title{
UN RESEAU DE FOSSÉS DÉFENSIFS AUX ORIGINES DE LYON
}

\author{
par Bernard MANDY avec la collaboration de Martine GENIN, \\ Catherine GODARD, Sophie KRAUSZ, Gérard SANDOZ, Philippe THIRION
}

La question des origines de Lyon et de l'implantation de la colonie romaine bien que fort débattue depuis longtemps reste ouverte. Faute d'arguments irréfutables, les solutions proposées sont régulièrement remises au cause ${ }^{1}$. La découverte au point culminant de la ville antique d'un réseau de fossés antérieurs à l'urbanisation relance évidemment le débat. Il s'agit, il faut le préciser, des premières traces tangibles remontant aux origines de la ville qui aient jamais été découvertes sur le site de la future agglomération ${ }^{2}$. De ce fait, il convient d'abor-

1 Le débat porte sur l'interprétation du texte de Dion Cassius (XLVI, 50) qui relate cet événement; il concerne à la fois, les circonstances de la fondation 'qualité des gens chassés de Vienne, date de leur départ), le statut de la colonie et son implantation par rapport au confluent. On retiendra essentiellement: P. Wuilleumier, Lyon, métropole des Gaules, 1953, p. 12 ; - A. Chagny, La fondation de Lyon el le souvenir de Munatius Plancus, Lyon, s.d., p. 21-35; - A. Audin, Essai sur la topographie de Lugdunum, 1956, p. 26-27 et plus récemment, Lyon miroir de Rome dar's les Gaules, 1965, p. 2528 ; - M. Rambaud, L'origine de la colonie de Lugdunum, Comptes Rendus de l'Académie des Inscriptions et Belles-Lettres, 1964 , p. 252-277; - A. Desbat et S. Walker, Le problème des origines de Lyon, dans Récentes recherches en archéologie gallo-romaine et paléochretienne sur Lyon et sa région, British Archeological Report, série 108, 1981, p. 29-53; P. Thollard, Strabon, Lyon, Vienne et les Ségusiaves, Revue Archéologique de Narbonnaise, XVII, 1984, p. 115-122; Chr. Goudineau, Note sur la fondation de Lyon, Gallia, 44, 1986, p. 171-173; - Origine et formation de Lugdunum, les découvertes récentes, Actes du séminaire de la Direction des Antiquités Hisloriques Rhône-Alpes, sous la direction de Chr. Goudineau (à paraître), Rapports archéologiques préliminaires de la région Rhône-Alpes où certains aspects de cette synthèse seront développés.

2 A. Desbat et S. Walker, op. cit. p. 33, ont bien montré l'inconsistance des arguments avancés par J. Chauffin der avec prudence cette occupation initiale, sans éluder le problème historique qui l'accompagne, mais en oubliant un instant les théories souvent spécieuses qui se sont développées à son propos. C'est pourquoi nous nous attacherons d'abord à décrire soigneusement les vestiges afin de préciser s'il s'agissait d'une installation cultuelle ou profane dans un contexte civil ou militaire, en milieu indigène ou romain, avant de poser le délicat problème de leur datation absolue.

C'est au cours d'une fouille de sauvetage programmé dans la propriété de la congrégation du Verbe Incarné, que les tronçons de fossés ont été découverts. Ce tènement, limité par les rues P.-M.Jaricot à l'o. et R.-Radisson au s., formait avant la mise en chantier du projet immobilier, cause du sauvetage, un quadrilatère d'environ 4 ha entièrement clos de murs dont seule la bande jouxtant la rue Radisson était bâtie. Topographiquement, la position de ce clos est intéressante puisqu'il est situé sur le point culminant de la ville antique. Il occupe plus précisément le bord oriental du plateau de La Sarra à l'endroit où une dépression naturelle, le vallon de La Chana, le sépare de l'éperon de Fourvière proprement dit (fig. 1A).

et $\mathrm{A}$. Audin qui ont cru déceler une occupation indigène dans un sondage au-dessus du théâtre. Cf. J. Chauffris et A. Audin, Essai de stratigraphie archéologique à Fourvière, Revue Archéologique de l'Est, no 23, 1972, p. 89-95; A. Audin, Dossier des fouilles du sanctuaire lyonnais de Cybèle et de ses abords. Annexe II, le site primitif, Gallia 43, 1985, p. 124-126. 
Fig. 1 - A. Plan topographique du site de Lugdunum.

1, Clos du Verbe Incarné;

2 , rue Le Chatelier.

B. Situation des fossés dans le clos du Verbe Incarné

(le grisé indique l'urbanisme antique).
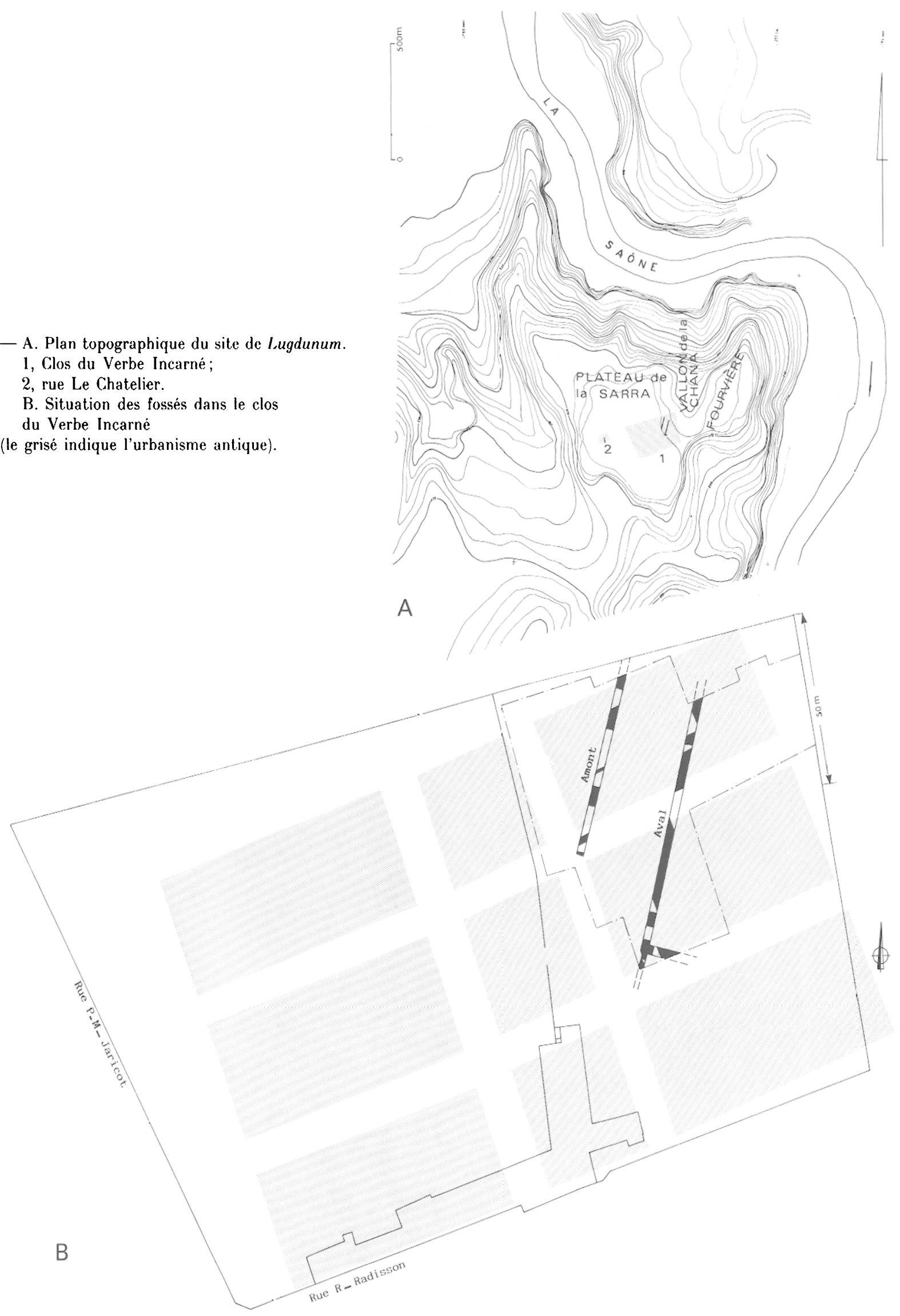


\section{MORPHOLOGIE}

Les traces de fossés n'ont été découvertes que sur le versant du vallon, c'est-à-dire dans la moitié orientale de la propriété ${ }^{3}$. Elles se détachent plus ou moins nettement sur le fond ocre du substrat nature ${ }^{4}$ et s'organisent selon une orientation très différente de celle des aménagements postérieurs (fig. 1B).

Les divers segments retrouvés appartiennent à deux ouvrages dont les axes, distants de $26 \mathrm{~m}$ sont parallèles à $2^{\circ}$ près. Ils sont perpendiculaires à la plus forte pente du terrain qui présente à cet endroit une déclivité de $14 \%$ vers l'e.

\section{Ouvrage amont}

Le fossé amont, repéré dès 1981 , a été recoupé six fois, sur une distance totale de près de $60 \mathrm{~m}$. Son axe, rectiligne, forme un angle de $13^{\circ}$ e. par rapport au n. et il suit une pente moyenne de $4,5 \%$. Le tronçon le plus méridional présente une interruption nette (fig. 2). Le caractère volontaire et construit de cette extrémité ne fait aucun doute étant donné sa régularité et le soin apporté à sa réalisation. Au-delà, l'absence du fossé a été constatée sur près de $4 \mathrm{~m}$, c'est-à-dire jusqu'en limite de fouille; il est toutefois impossible de préciser si le tracé ne reprenait pas plus loin.

Le profil est complet dans le tronçon le mieux

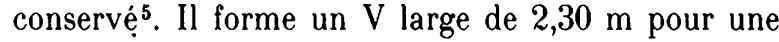
profondeur de $1,50 \mathrm{~m}$. Les parois lisses et très régulières, ne présentent aucune trace d'outil ni de ravinement. Elles se terminent par une étroite rigole, de 10 à $15 \mathrm{~cm}$ de large, au fond de laquelle se détachent des négatifs de piquets qui, sur la longueur du tronçon, sont régulièrement espacés de 25 à $30 \mathrm{~cm}$ (fig. 3). Précisons que ce profil aigu et régulier est également caractéristique des autres segments repérés.

3 Ils n'ont été mis en évidence qu'à l'intérieur du périmètre préalablement déterminé par le sauvetage programmé. Les sondages indispensables à toute recherche complémentaire de tracé présentaient un caractère trop aléatoire quant au résultat pour être entrepris.

4 La roche mère est ici constituée d'un limon de couleur ocre et d'origine éolienne, le lœss; elle est par endroits recouverte d'une couche de $0,20 \mathrm{~m}$ à $0,30 \mathrm{~m}$ d'épaisseur de même constitution mais de teinte rouge, il s'agit du lehm qui résulte de l'altération par pédogenèse du lœess qui signale la proximité du paléosol originel.

5 La preuve en est donnée par divers débordements de la couche de comblement sur les deux rives.

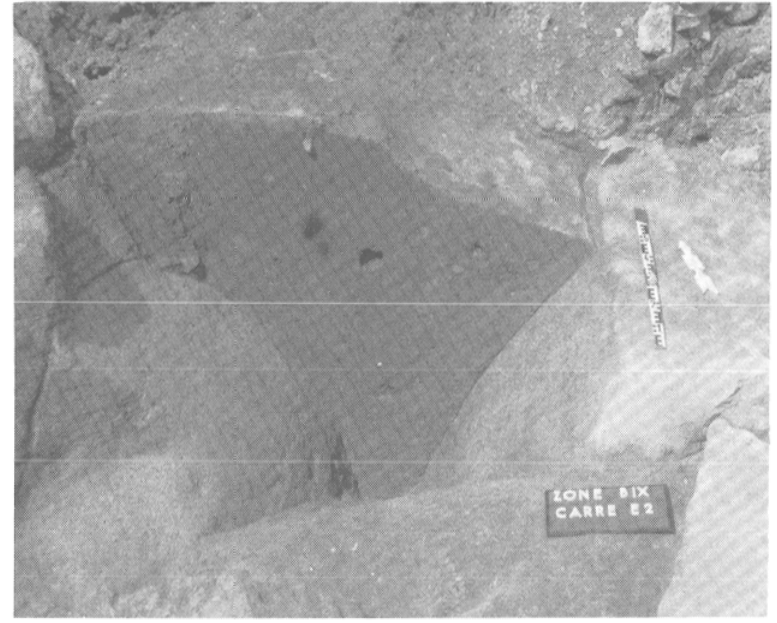

Fig. 2 - Extrémité sud du fossé amont.

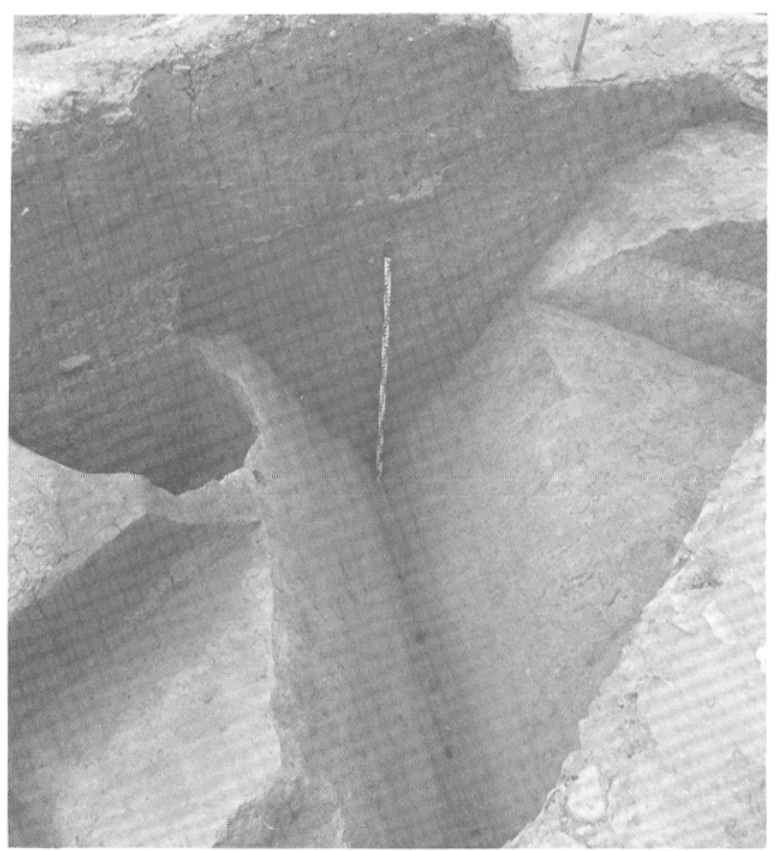

Fig. $3-$ Vue du fossé amont.

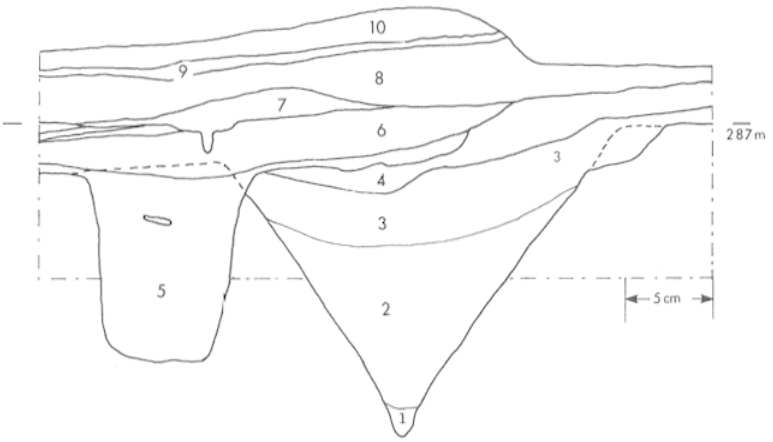

Fig. 4 - Coupe stratigraphique du fossé amont. 1-3: comblement du fossé; 4: couche colluvionnée (abandon); 5-10: occupations postérieures. 


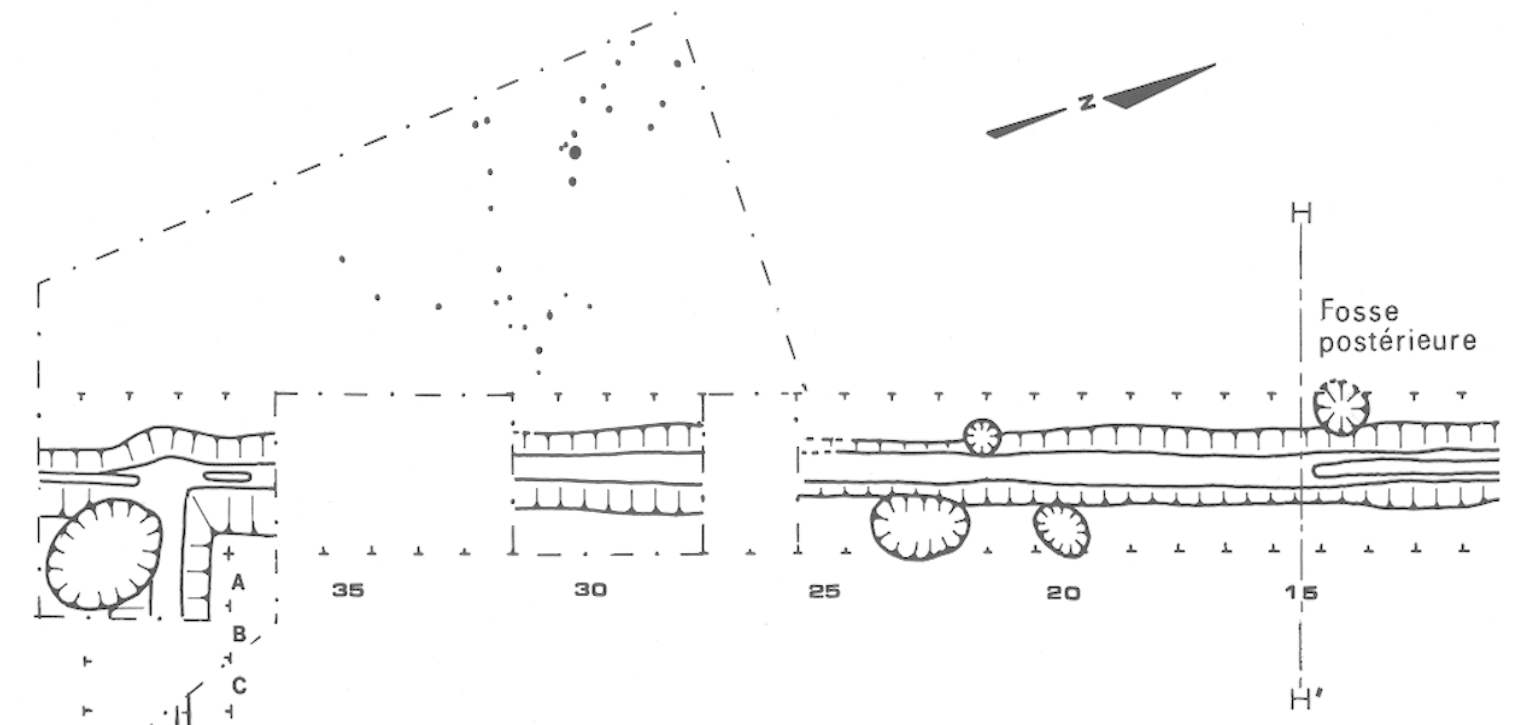

Fig. 5 - A gauche, plan partiel du fossé aval; à droite, détail des segments 1 à 15 .

Le comblement consiste en un mélange de lehm et de lœess. Bien qu'il n'y ait pas à proprement parler stratification du remplissage, nous remarquons toutefois l'existence de lits d'épandage de teintes légèrement différentes dont l'inclinaison vers l'aval suggère un comblement depuis le bord amont (fig. 4). Nous ne constatons ni classement granulométrique des éléments ni trace de dépôt colluvionné; les macro-éléments, à savoir des galets (dont certains sont rubéfiés) et le mobilier archéologique (tessons d'amphores et ossements), restent en nombre restreint et ne présentent pas d'orientation particulière.

Trois points se dégagent de ces constatations :

- d'une part, l'absence de signes d'érosion sur les parois et de traces de sédimentation sur le fond laisse présumer que le fossé n'est pas resté longtemps en usage ;

- d'autre part les caractéristiques du comblement indiquent que ce fossé a été rebouché de main d'homme, probablement avec ses propres déblais;

- enfin, l'existence, immédiatement au-dessus du comblement, d'une série de dépôts limoneux, comportant de nombreuses empreintes végétales, est le témoin incontestable d'une période d'abandon.

\section{Ouvrage aval}

Ce deuxième fossé, identifié dès 1983 est maintenant connu sur plus de $70 \mathrm{~m}^{6}$. Il suit un axe de $15^{\circ} \mathrm{e}$.

$642 \mathrm{~m}$ ont été fouillés à partir d'un maillage spécifique, constitué de segments de $1 \mathrm{~m}$ de large, disposés perpendiculairement à l'axe du fossé, afin de mieux appréhender le processus de comblement et les accumulations de mobilier qui paraissait particulièrement abondant dans ce secteur. par rapport au n. et une pente moyenne de l'ordre de $5 \%$, il est donc quasiment parallèle au précédent. Il présente à son extrémité $s$. un embranchement aval, et ce concurremment avec un prolongement dans son axe (fig. 1B). Cette ramification qui forme un angle d'environ $90^{\circ}$ avec le reste du fossé, n'a été suivie que sur quelques mètres en raison d'une limite de fouille. Bien que de larges décapages des abords aient été exécutés en amont et en aval pour rechercher d'éventuelles structures associées, aucune trace susceptible d'être en relation avec le fossé n'a été dégagée, hormis quelques trous de piquets apparemment inorganisés, sur le bord o. à hauteur des segments 28 à 32 .

L'étude du tronçon méridional montre une grande similitude entre le fossé principal et son embranchement aval : ils possèdent le même profil en $U$ dont le fond plat varie entre $0,50 \mathrm{~m}$ et $0,70 \mathrm{~m}$ de largeur. En revanche, le prolongement s. présente un profil en $\mathrm{V}$ de faible dimension $(1,30 \mathrm{~m} \times 0,60 \mathrm{~m})$ qui contraste avec celui des deux branches précédentes. Par le fait, il constitue une sorte d' "antenne» audelà de l'angle qu'elles forment (fig. 6). Les parois des deux branches principales sont irrégulières et bosselées; elles comportent par endroits des traces sinueuses plus ou moins verticales qui évoquent plutôt de petites ravines que des traces d'outils.

Comme dans l'ouvrage amont, une rigole médiane, variant de 15 à $30 \mathrm{~cm}$ de largeur, et de profil semi-circulaire garnit le fond de ces fossés. Son tracé, continu dans toute la moitié n. s'interrompt sur $1 \mathrm{~m}$ à hauteur du segment 4 puis à nouveau des segments 15 à 39 . Un grand nombre de négatifs de piquets a été découvert, mais uniquement dans les 


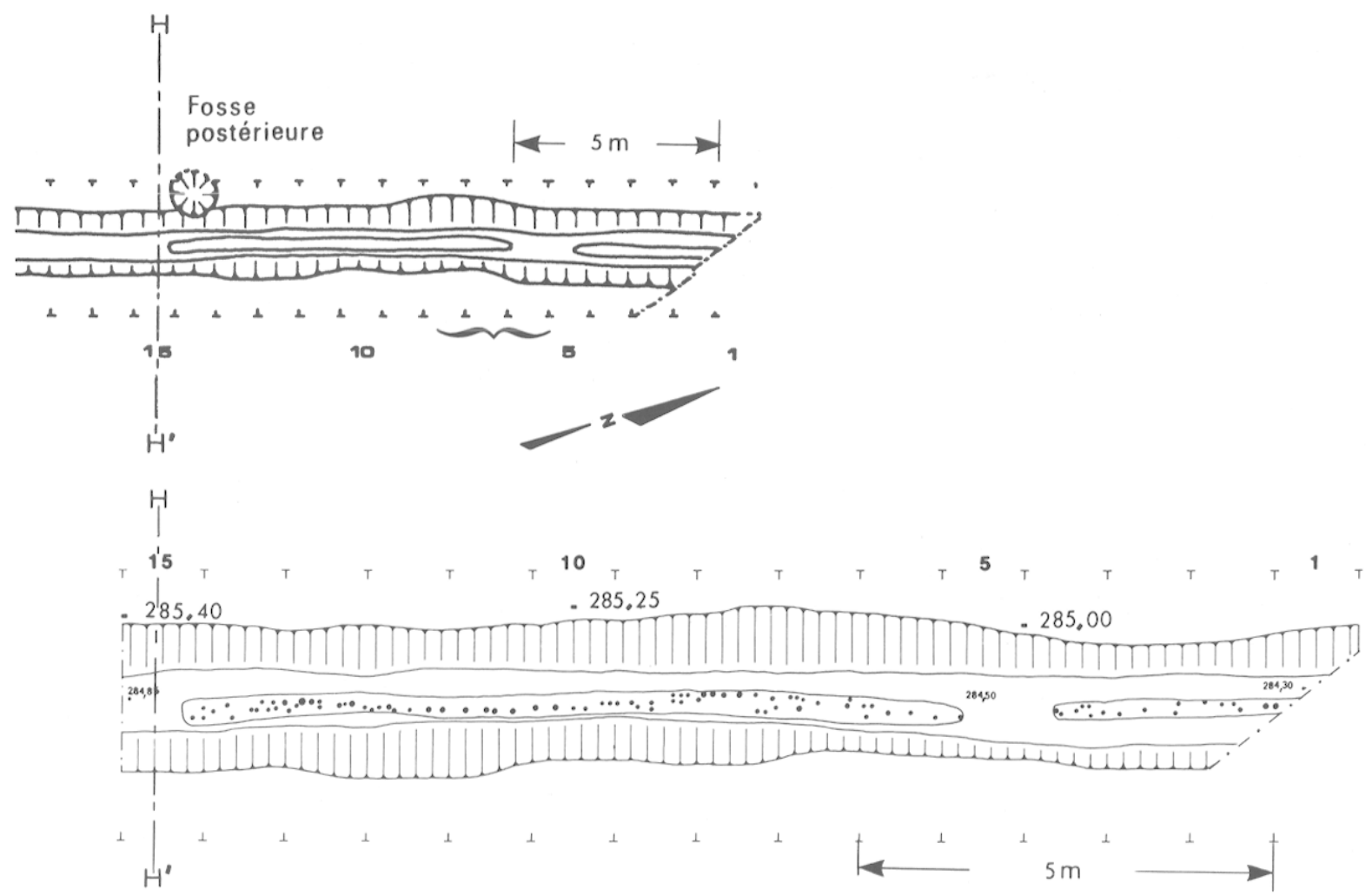

portions munies de rigoles. De taille réduite (4 à $6 \mathrm{~cm}$ de section pour 5 à $10 \mathrm{~cm}$ de profondeur), ils étaient disposés sans ordonnance (fig. 5).

Le remblaiement du prolongement $\mathrm{s}^{2}$, constitué d'un matériau homogène, est manifestement antérieur à celui des deux branches principales. La stratigraphie de ces dernières, plus complexe que celle du fossé amont, a fait l'objet d'une analyse minutieuse et d'une étude de laboratoire ${ }^{8}$; elle peut se résumer en sept couches significatives dont la plupart sont intermittentes (fig. 6) :

\section{Couche 1}

Couche de terre brune, homogène et sans mobilier, comblant la rigole. Son aspect tassé et l'absence de dépôts colluvionnés indiquent qu'il pourrait s'agir

7 Le comblement de cette partie est toutefois de nature très semblable à celui du reste du fossé, c'est pourquoi nous l'avons considéré comme une simple phase dans le remblaiement général.

8 Cette étude a porté sur une carotte de $20 \mathrm{~cm}$ de section prélevée dans le segment 20 . Elle a été effectuée par M. Dubar, chargé de recherche au C.R.A. de Sophia Antipolis et comprenait des analyses sédimentologiques, malacologiques et palynologiques. d'un comblement volontaire servant à caler les piquets, bien que la trace de ces derniers n'y ait pas été retrouvée. La disparition des négatifs de piquets dans cette couche s'expliquerait par une récupération systématique de ceux-ci lors de l'abandon.

\section{COUCHE 2}

Mince pellicule de terre jaune présente sur toute la longueur fouillée, de granulométrie très fine et sans mobilier. Elle tapisse le fond proprement dit du fossé et correspond peut-être à un dépôt naturel.

\section{COUCHE 3}

Série de strates, difficilement séparables à la fouille où alternent de fines pellicules de cendres avec une terre charbonneuse, meuble, contenant de nombreux ossements très fragmentés dont beaucoup présentent des traces de calcination. Cette couche qui comporte également de nombreux restes de soles de foyer et de tessons brûlés ainsi que des galets rubéfiés résulte probablement de la vidange de foyers. Sa position sur le bord oriental montre clairement qu'elle a été déversée depuis l'aval.

\section{Couche 4}

Couche d'épaisseur variable située sur la paroi occidentale du fossé et composée d'une terre brune mêlée de nodules de lœss. 

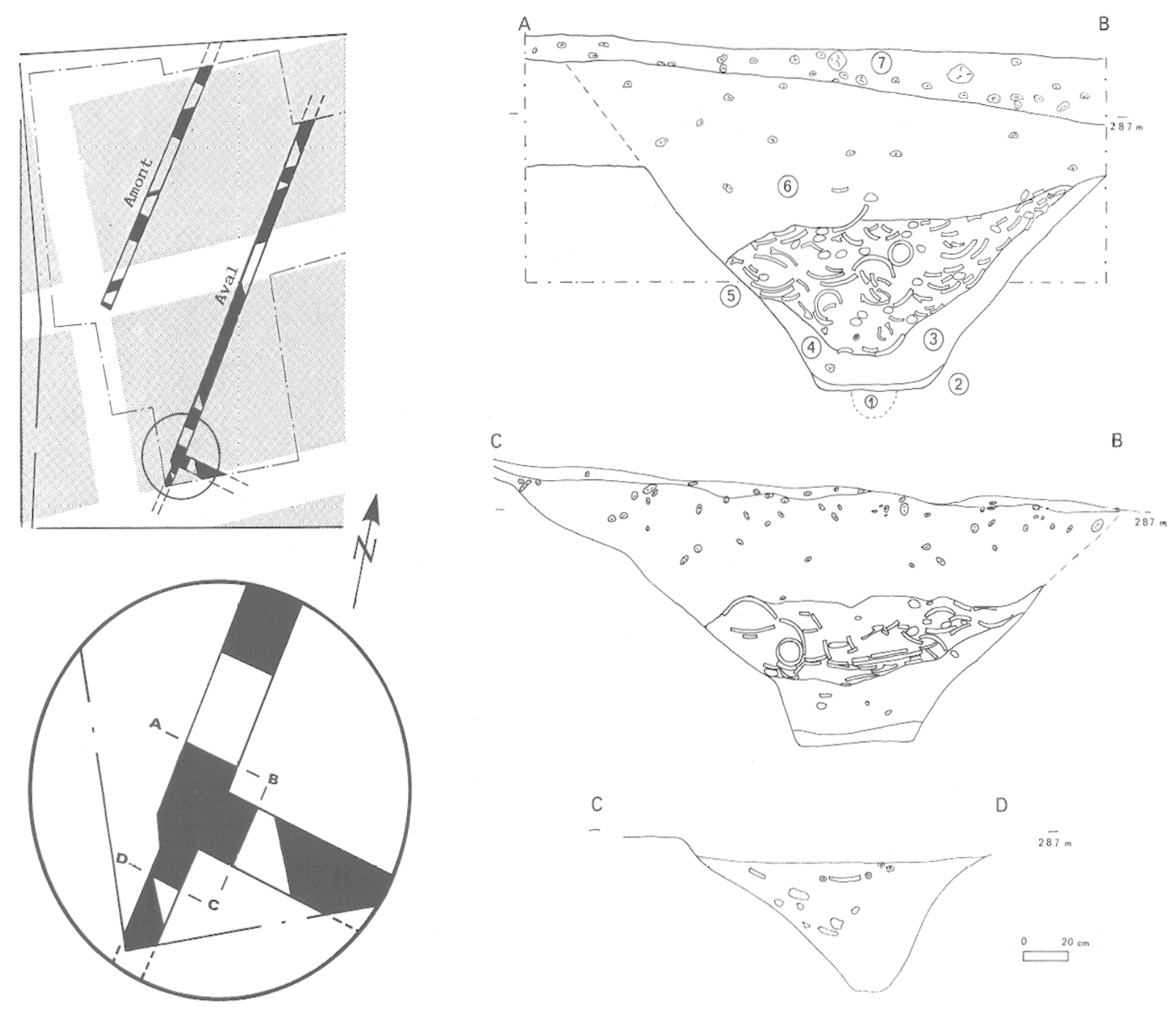

Fig. 6 - Coupes stratigraphiques comparées des trois branches du fossé aval.

\section{COUCHE 5}

Dépôt abondant de fragments d'amphores et d'ossements équivalent à une véritable couche qui présente un maximum de densité dans les carrés 15 à 39 . Là encore la disposition du matériel suggère un déversement depuis l'aval (fig. 7).

\section{COUCHE 6}

Couche de nature très voisine du lehm environnant, de couleur ocre, contenant amphores, ossements et de nombreux galets. Elle constitue l'essentiel du remplissage, et son origine anthropique ne fait aucun doute.

\section{Couche 7}

Préservée uniquement à l'extrémité méridionale, elle scelle le comblement proprement dit des fossés. Elle est formée de fines strates colluvionnées qui suivent la pente naturelle du terrain. De nombreuses traces de végétaux et des petits galets y sont visibles.

Le comblement terminal (couche 6 ) pourrait se distinguer d'une phase préalable, correspondant aux couches 3 et 5 , particulièrement riches en matériel : celles-ci ont manifestement été déversées depuis le bord aval ; celui-là présente en revanche un épandage entrecroisé plus difficile à interpréter. Mais, la dispersion verticale des fragments de plusieurs vases montre que le comblement général a suivi de peu la phase de dépôt.

Bien qu'effectuée sur un échantillon très ponctuel, l'étude sédimentologique vient à l'appui de ces données stratigraphiques. Elle confirme que la limite séparant le comblement du substrat lœssique ne montrait aucun phénomène de ravinement ou de 

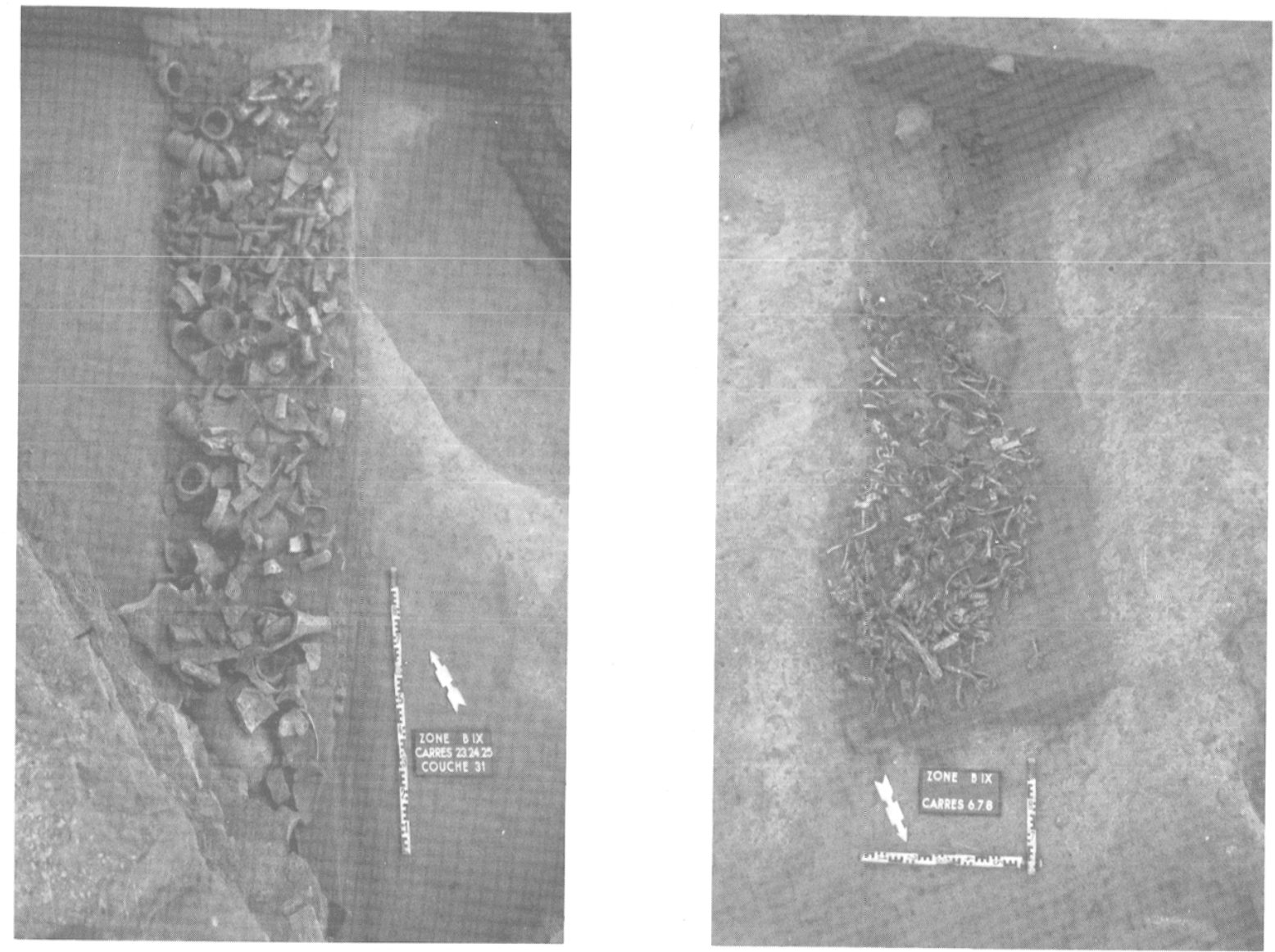

Fig. 7 - Accumulation d'amphores et d'ossements dans le fossé aval (couche 5).

compactage. De plus, l'examen du matériau de comblement atteste qu'il provient de l'environnement immédiat du fossé et surtout qu'aucune stratification naturelle n'existe dans le remplissage ${ }^{9}$. Ce dernier point confirme, s'il en était besoin, que le colmatage du fossé a suivi de peu son creusement.

\section{MATÉRIEL}

Les quelques ossements et la poignée de tessons recueillis dans le fossé amont ${ }^{10}$ contrastent singulièrement avec l'abondance de mobilier du fossé aval. Bien qu'ils soient en nombre trop limité pour se prêter à une étude, ils permettent néanmoins d'établir une certaine parenté entre les deux ensembles.

9 La carotte ayant été prélevée sur le bord amont du fossé pour pouvoir étudier la jonction entre comblement et terrain naturel, l'analyse n'a pu tenir compte des couches tapissant le fond.

10 Ce mobilier se compose de 38 restes osseux, de 18 fragments d'amphores, 52 tessons de céramiques communes et de 2 fragments en fer dont une sorte d'anneau.
Le matériel du fossé aval présente une composition tout à fait inhabituelle qui ne constitue pas le moindre des intérêts de cette découverte. Formé à $96,5 \%$ d'amphores et d'ossements, il dénote en effet une occupation d'un caractère assez particulier (fig. 8). C'est pourquoi nous traiterons ces deux ensembles, généralement considérés comme secondaires et renvoyés en annexe, avant même d'étudier le reste du matériel dont l'intérêt essentiel réside dans l'écart chronologique qu'il révèle.

Nous sommes obligés d'évoquer d'abord les quelques restes humains découverts au milieu des tessons d'amphores et qui détonnent quelque peu dans cet ensemble. Ils se résument à une tête de femme âgée de 25 à 40 ans dont le reste du squelette n'a pas été retrouvé ${ }^{11}$. Mais la mandibule et deux vertèbres cervicales étaient encore en connexion anatomique avec le crâne, ce qui ne peut guère s'expliquer que par une décapitation.

11 L'étude ostéologique a été conduite par B. Bizot I.T.A. à la Direction des Antiquités Historiques d'Aquitaine. 


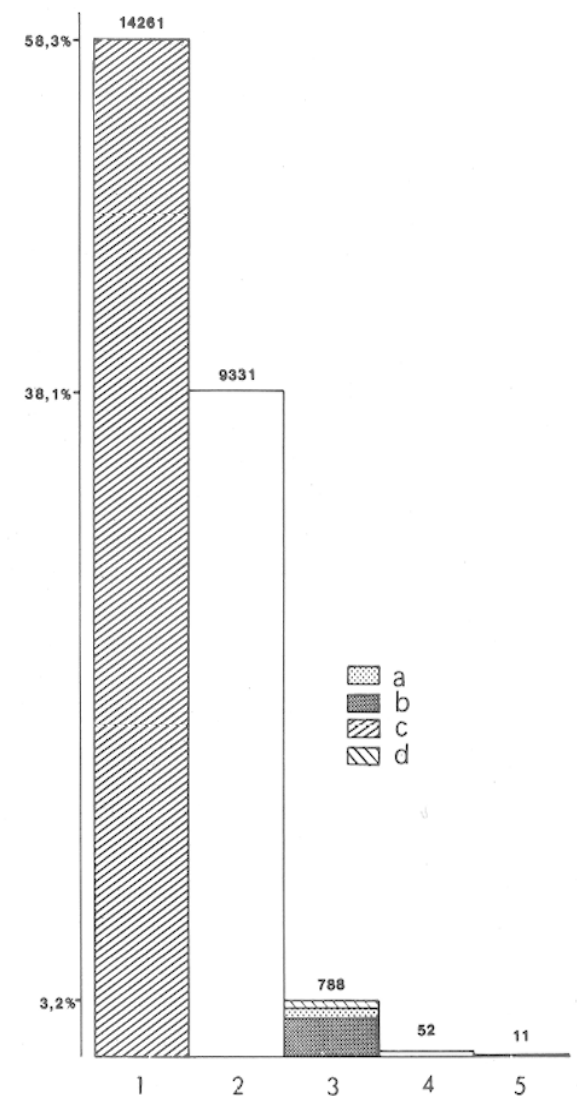

Fig. 8 - Histogramme du matériel du fossé aval.

1 , amphores ; 2 , ossements; 3 , céramiques diverses ; 4 , métal ; 5 , divers.

$a$, céramiques tournées indigènes; $b$, céramiques non tournées indigènes; $\mathrm{c}$, amphores importees; $\mathrm{d}$, autres céramiques importées.

\section{La FAUNe}

Les résultats présentés ici ne constituent que les préliminaires de l'étude menée par S. Krausz sur la faune. Il s'agit essentiellement de données générales proposant une image de la composition du lot, tant du point de vue des espèces que des parties anatomiques représentées. Les comptages permettent également de jeter les bases d'une interprétation qui devra toutefois être complétée par des études plus détaillées.

L'ensemble des restes représente 9331 ossements d'animaux dont $68 \%$ ont été identifiés. Cette forte proportion est due à l'excellente conservation du matériel constellé de concrétions calcaires qui ont contribué à sa préservation. Grâce à ces concrétions, de nombreux segments anatomiques en connexion ont été observés (notamment des pattes de porc). L'étude a, en outre, montré qu'il n'existe pas de différence significative entre les différentes couches du remplissage, constatation qui pourrait étayer l'hypothèse d'un comblement rapide.
Le premier point important de cette étude est la sur-représentation du porc qui constitue en effet à lui seul $94 \%$ des restes. De plus, les pièces consommées (parties d'os longs, côtes) et les pièces non consommées (vertèbres, dentures, extrémités des pattes) coexistent dans les mêmes proportions, c'est-à-dire qu'il existe aussi bien les rejets des activités bouchères que de la consommation alimentaire ellemême. L'analyse ultérieure, par âge et par sexe s'efforcera d'établir si la composition de ce cheptel reflète le mode habituel de gestion d'un troupeau ou plutôt une sélection d'animaux de boucherie. Les autres espèces (bœuf, cheval, mouton, chèvre, lapin, cerf, chien et renard) représentent $6 \%$ du nombre total de restes. Ceci indique une activité de chasse quasi inexistante et un élevage très réduit d'animaux de scrvice.

En ce qui concerne l'analyse biométrique, douze pièces anatomiques provenant de porcs, ont été mesurées; elles ont permis d'estimer douze hauteurs au garrot : les tailles vont de 68 à $80 \mathrm{~cm}$ avec une moyenne de $72,5 \mathrm{~cm}$ qui s'inscrit parfaitement dans la taille des porcs connus à La Tène finale. Pour les équidés, nous avons pu estimer deux tailles (respectivement 135 et $139 \mathrm{~cm}$ au garrot); elles paraissent nettement supérieures aux estimations habituellement proposées pour les chevaux indigènes de cette période ${ }^{12}$.

Ainsi les premiers résultats de cette étude donnent l'image d'une occupation originale qui diffère grandement de celles des sites d'habitats contemporains.

\section{LES AMPHORES}

Dans le fossé aval, 14261 fragments d'amphores ont été retrouvés. L'étude d'une telle quantité de matériel pose un réel problème. Nous avons choisi d'évaluer le nombre minimum de vases à partir des éléments d'identification les plus sûrs à savoir lèvres et fonds, sans chercher, faute de temps, à reconstituer systématiquement les formes. On dénombre 272 lèvres différentes pour seulement 96 fonds, discordance qui pose d'emblée le problème du mode de formation du gisement.

12 Ces hauteurs ont été déterminées à partir de la dimension des os longs multipliée par les coefficients établis par Kieswalter (1888). On peut les comparer aux découvertes de Breisach-am-Rhein (All.) $120 \mathrm{~cm}$; Manching (All.) $124 \mathrm{~cm}$; Levroux (Indre) $126 \mathrm{~cm}$; Beauvais (Oise) $127 \mathrm{~cm}$. 
Grâce à la méthode de prélèvement mise en place en $1985^{13}$, le remontage systématique des 172 lèvres issues des segments 1 à 32 a permis de constater une faible dispersion horizontale des fragments les constituant, ce qui laisse supposer qu'elles se sont brisées sur place. De plus, le grand nombre d'opercules conservés, dont une douzaine était encore dans les cols, indique de façon évidente que ces cols ont été séparés du reste du récipient avant d'en vider le contenu. Des traces de décolletage sont d'ailleurs bien visibles au niveau de certains cols. Cette opération fut sans doute à l'origine d'une dispersion sélective des deux parties d'amphore : ceci peut expliquer le déficit en fonds dans cette portion du fossé.

Une analyse physico-chimique des pâtes effectuée par l'UKA $3^{14}$ sur 50 échantillons choisis de façon aléatoire, a mis en évidence que $80 \%$ des pâtes se répartissent en 6 groupes, tous d'origine italique : deux sont étrusques (gr. 1 et 3 ), deux campaniens (gr. 4 et 6), le cinquième (gr. 5) provient d'une région volcanique qui reste à identifier, quant au dernier (gr. 2) sa région d'origine n'est pas connue (fig. 9).

L'examen à la loupe binoculaire d'échantillons prélevés sur les 222 lèvres non analysées confirme cette faible disparité d'origine puisque, excepté $12 \%$ d'inclassables, elles se répartissent dans les six groupes de référence et trois groupes satellites ${ }^{\mathbf{1 5}}$. L'étude actuellement menée par M. Genin montre d'ores et déjà une nette corrélation entre types de lèvres et groupes de pâtes notamment pour les groupes $2,3,6$.

L'extraordinaire homogénéité des groupes 3 et 6 tant par la composition des pâtes que par la morphologie des lèvres ne laisse aucun doute sur l'origine de ces produits : le groupe 3 correspond aux productions de Sestius à Cosa et le groupe 6 à celle d'Eumachie à Pompéi. La cohérence de deux autres groupes (gr. 1 et 2) évoque également des lots de provenance très localisée sans qu'on connaisse toutefois précisément les officines. Le groupe 4, spécifique de Campanie, présente en revanche une apparence trop hétéroclite pour correspondre à une production

13 Cf. supra, note 6, p. 52.

14 Nous remercions M. Picon et le laboratoire de céramologie installé à la Maison de l'Orient (URA 3 du Centre de Recherches Archéologiques) de s'être gracieusement chargés de cette étude.

15 Le caractère empirique de ce type d'analyse a conduit à établir par précaution 3 groupes supplémentaires (respectivement proches des groupes 1, 2 et 4) dont seule l'analyse physico-chimique pourra ètablir le bien-fondé. unique; il rend plutôt compte de la multiplicité des ateliers de cette région.

En regard de la quantité de matériel, cette faible diversité de provenance est exceptionnelle; seul un approvisionnement de courte durée peut l'expliquer. Mais, cette diversité d'origines augmente compte tenu des amphores inclassables. Ceci nécessite de nuancer la notion de brièveté, sinon pour la constitution du dépôt lui-même, du moins pour l'approvisionnement bien que nous ignorions tout des conditions dans lesquelles il s'est effectué.

La rapiditè de formation du dépôt soulève indirectement le problème de la typologie traditionnelle des Dressel 1. Sans entrer dans le détail, il est clair qu'il n'y a pas d'adéquation, bien au contraire, entre les caractéristiques des groupes qu'on vient de déterminer et celles généralement proposées pour définir les Dr. 1A, B et C. Certes le groupe Eumachie, d'ailleurs très minoritaire, recouvre sans ambiguité le type Dr. 1C. Mais pour le reste, les groupes même les plus homogènes, tel que celui de Sestius par exemple, participeraient à la fois des Dr. 1A et $1 \mathrm{~B}$ si on s'en tient aux critères généralement retenus pour les lèvres ${ }^{16}$. Ce constat de carence de la typologie traditionnelle conduit évidemment à émettre les plus vives réserves quant à la datation qu'elle pourrait induire.

\section{LES AUTRES MOBILIERS}

\section{CÉRAMIQUES}

Outre les quelques 15000 tessons d'amphores, le fossé aval a livré 788 fragments de céramiques diverses. Après recollage, 52 vases ${ }^{17}$, répartis en quatre grandes catégories, ont été dénombrés.

\section{La céramique non tournée}

Elle représente $70 \%$ des fragments et $55 \%$ des vases identifiables. La majorité des récipients est montée au colombin avec une argile contenant de

16 En effet, la hauteur de plusieurs lèvres de ce groupe qui typologiquement appartient aux Dr. 1A est en contradiction avec l'affirmation de: A. Tchernia, Le vin de l'Italie romaine. Essai d'histoire économique d'après les amphores, Bibliothèque de l'Ecole Française d'Athènes et de Rome 261, 1986, p. 313 «toutes les amphores dont la lèvre fait plus de $5,5 \mathrm{~cm}$ appartiennent à cette forme (Dr. 1B) où à des Dr. 1C".

17 Les comptages ont été effectués selon la méthode préconisée par P. et C. Arcelin. Cf. P. Arcelin et C. ArcelinPradelle, Un problème de méthode: choix des données quantitatives en céramologie, Documents d'Archéologie Méridionale, 4, 1981, p. 189-192. 


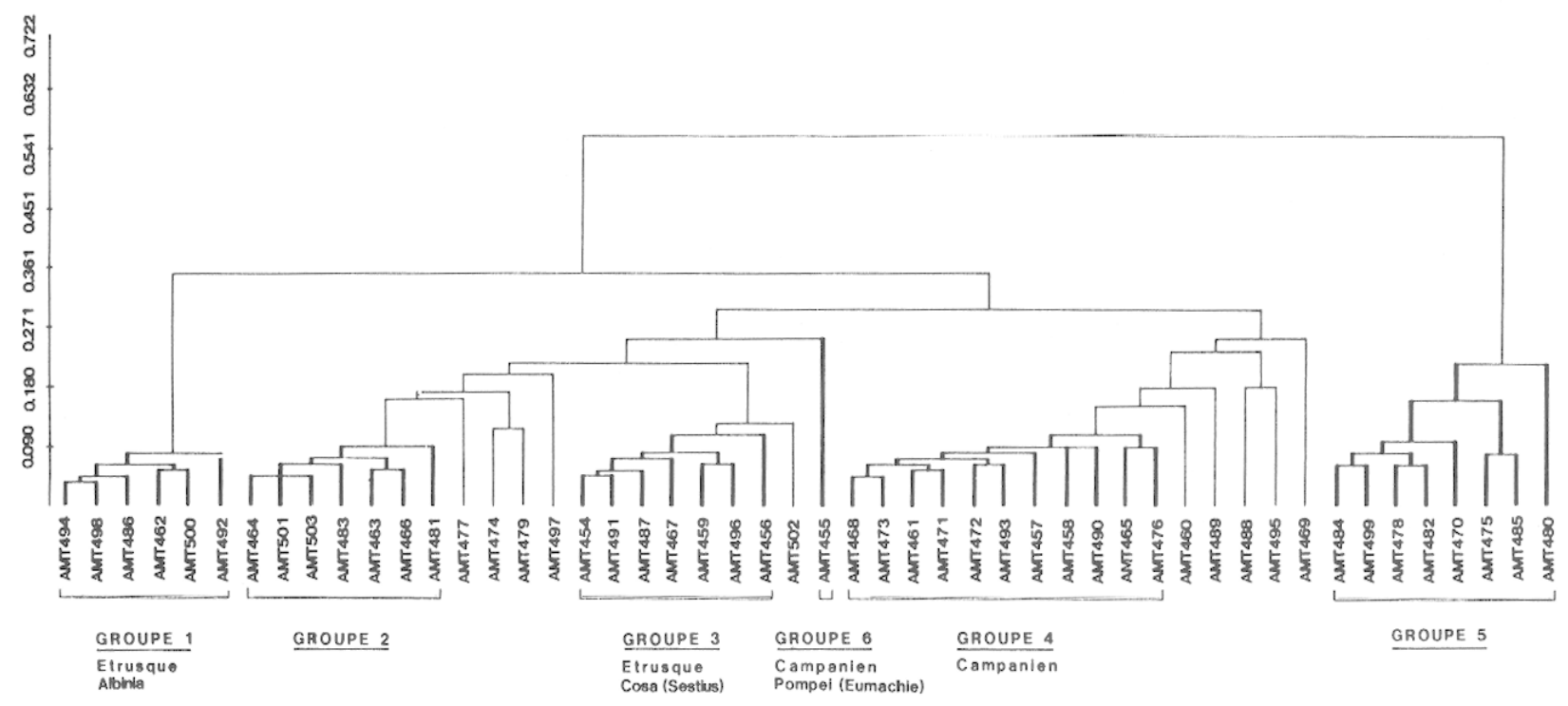

groupe 1
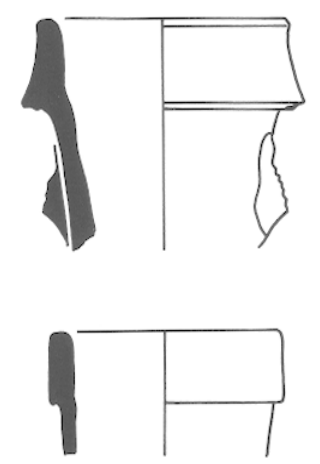

groupe 2
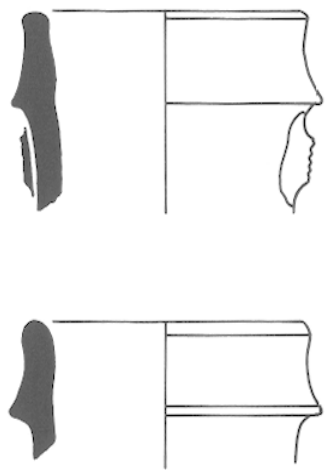

groupe 5

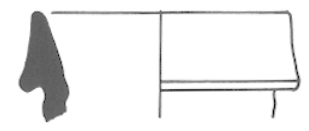

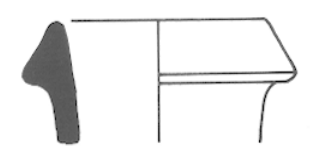

$0-5 \mathrm{~cm}$

groupe 4

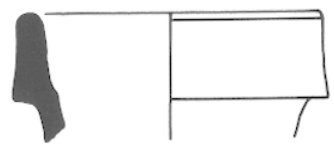

groupe 3
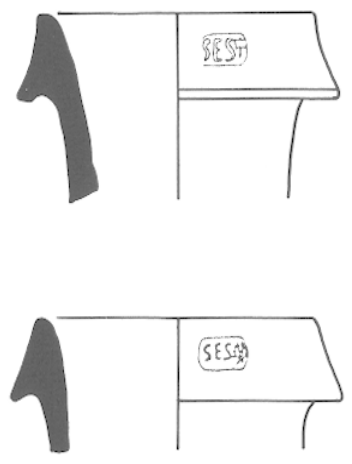

groupe 6

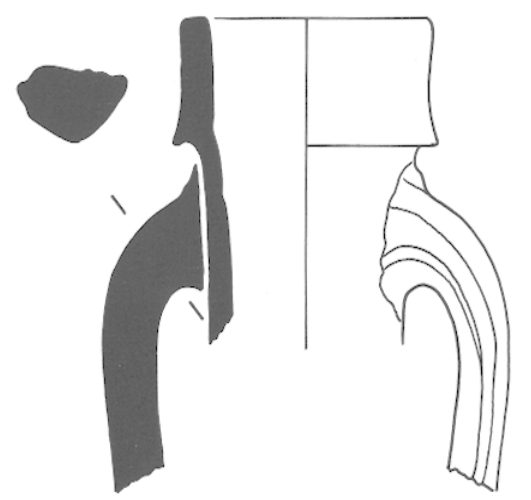

Fig. 9- Analyse de grappe el groupes méthodologiques correspondants. 

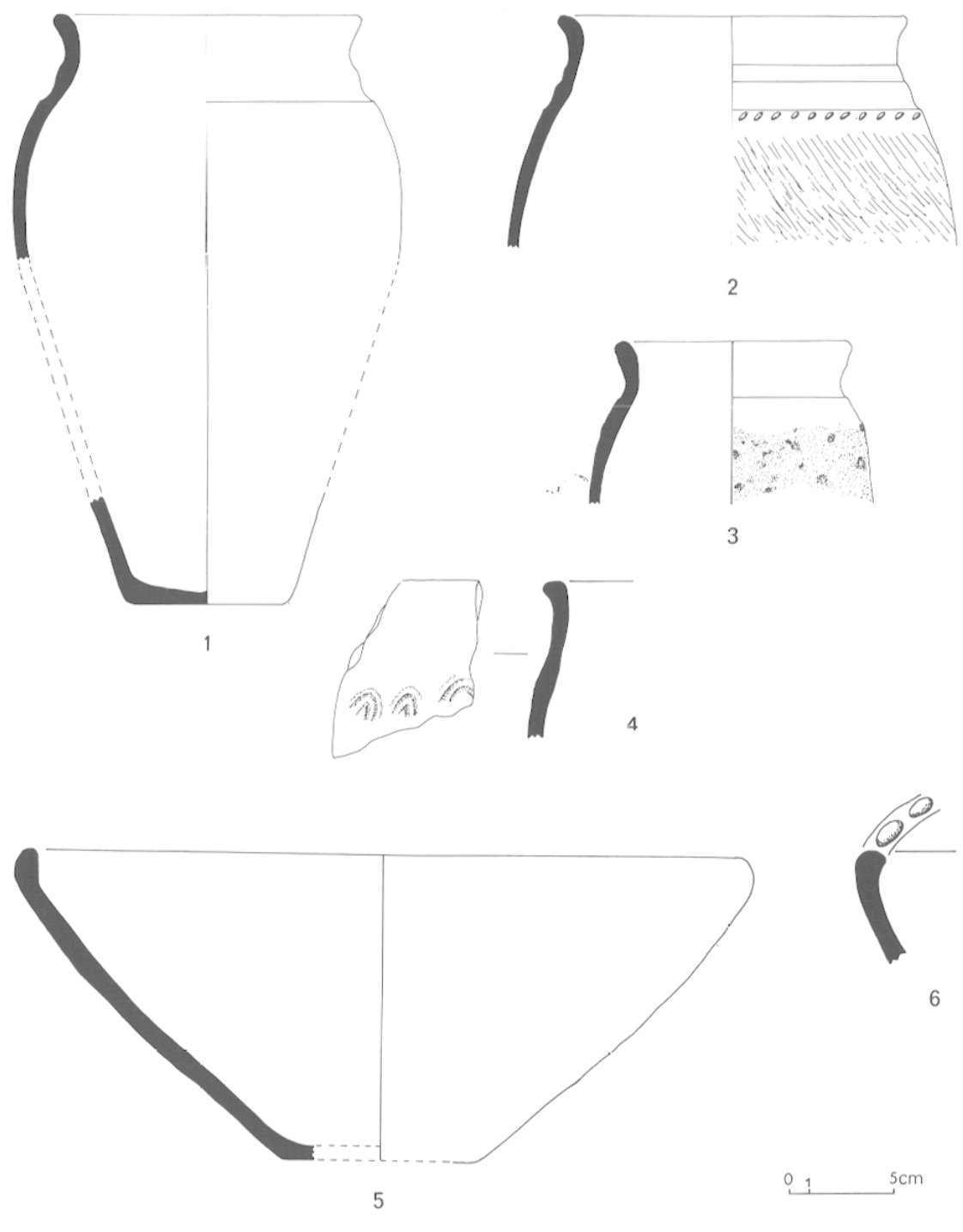

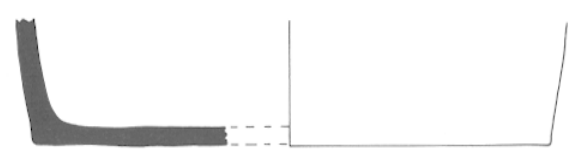

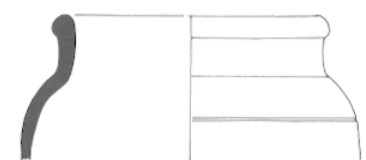

8

Fig. 10 - Céramiques diverses. 1-7, céramiques non tournées ; 8 , céramique demi-fine tournée.

nombreuses particules visibles à l'œil nu qui leur confèrent un aspect grossier. La cuisson en mode A primitif ${ }^{18}$ donne aux pâtes une teinte allant de l'ocre au noir.

Plus de la moitié des céramiques de cette catégorie, soit seize vases, correspond à une même forme de récipient haut, fermé, à panse ovoïde et

18 M. Picon, Introduction à l'élude technique des céramiques sigillées de Lezoux, 1973. fond plat. Les cols droits et lissés sur les deux faces comportent souvent une série de moulures irrégulières et se terminent par une lèvre évasée. Les décors sont rares : un exemplaire (fig. $10, \mathrm{n}^{\circ} 2$ ) présente une ligne de décors effectués au bâtonnet, un autre (fig. 10, $n^{0} 4$ ) une série d'arcatures estampées.

Les formes basses sont représentées par six écuelles à bord rentrant (fig. $10, \mathrm{n}^{\text {os }} 5$ et 6 ). Les parois sont droites ou légèrement convexes et les fonds plats. Deux sont décorés d'impressions digitées. Les diamètres d'ouverture sont compris entre 26 et $40 \mathrm{~cm}$. 
Six couvercles à bord droit, un fond plat de marmite cylindrique (fig. $10, \mathrm{n}^{0} 7$ ) d'un diamètre d'environ $23 \mathrm{~cm}$ sont également recensés.

\section{La céramique demi-fine}

Nous n'en avons retrouvé qu'un seul fragment qui provient d'un vase ovoïde à col droit et lèvre en bourrelet; le haut de la panse et le col sont lissés au brunissoir (fig. 10, $\mathrm{n}^{\circ} 8$ ). Cette forme tournée dans une argile à dégraissant de taille moyenne, est caractéristique de productions bien connues dans la Loire ${ }^{19}$.

\section{La céramique fine}

Le traitement de surface des vases permet de les subdiviser en deux sous-catégories :

- La céramique «lissée» qui représente $17 \%$ des vases et $10 \%$ de l'ensemble des fragments, est caractérisée par une pâte sans dégraissant apparent et une finition souvent très soignée. Cuite en mode $\mathrm{A}$ primitif comme la céramique non tournée, mais selon une technologie mieux maittrisée, elle présente en surface une teinte gris-noir qui résulte d'une fumigation en post-cuisson.

Pour les formes fermées, seulement deux vases à panse ovoïde sont dénombrés (fig. $11 ; \mathrm{n}^{\text {os }} 1$ et 2 ). Un vase $\left(n^{0} 1\right)$ est décoré de trois moulures. Les formes ouvertes rassemblent cinq récipients. Deux d'entre eux sont des jattes à profil en $\mathrm{S}$, col droit et lèvre évasée (fig. $11, n^{\circ s} 3$ et 4 ). Une petite coupe également à col droit s'apparente à la même forme quoique de dimensions plus réduites (fig. 11, $\mathrm{n}^{\circ} 6$ ). Un fragment de rebord évoque une coupe à marli qui s'inspire directement du répertoire des céramiques campaniennes (forme Lamb. 36) (fig. 11, $\mathrm{n}^{0} 5$ ). Un fond atteste l'existence d'une écuelle à bord rentrant.

- La céramique peinte qui constitue $11,5 \%$ des vases et $3 \%$ du nombre de fragments, regroupe des récipients en pâte fine de teinte beige-orangé, cuits selon un mode réducteur-oxydant dont la postcuisson a été très bien contrôlée.

Cinq des six fragments appartiennent à des vases dits "fuselés» que caractérise un corps élancé terminé par un bas de panse cintré et un fond étroit (fig. 11, nos 7 à 9). L'un des fragments $\left(\mathrm{n}^{\circ} 8\right)$ montre

19 Ce groupe de production correspond à un répertoire de formes très limité (vases ovoïdes). Pratiquement absent de Roanne, il est en revanche bien représenté sur les sites du Forez. Cf. M. VAginay et V. Guichard, L'habital gaulois de Feurs (Loire), fouilles récentes (1979-1981), Documents d'Archéologie Française (à paraître). Nous remercions les auteurs de nous avoir communiqué leur manuscrit. un décor géométrique formé de deux bandes accolées, l'une blanche, l'autre rouge à décor d'échelle horizontale brun. Le sixième correspond à un fond de vase dont la pâte rouge-orangé et la forme pourraient rappeler certains vases peints du Midi (fig. $\left.11, \mathrm{n}^{0} 10\right)^{20}$.

\section{La céramique importée}

Trois vases italiques à vernis noir ont été identifiés; deux sont caractéristiques de la Campanienne A : pâte dure, vernis fragile s'écaillant par plaques et à reflets métallescents; ils correspondent aux formes Lamb. 36 et Lamb. 27 (fig. 11, nos 12 et 14). Le troisième présente une pâte plus calcaire, un vernis mat et de bonne qualité; il s'agit d'une assiette en campanienne B de type Lamb. 5 (fig. 11, $\left.\mathrm{n}^{\circ} 13\right)$.

Cinq fragments de céramique à pâte claire d'origine méditerranéenne ont également été recensés. Il s'agit de fonds de cruches en pâte blanc-crème avec un pied annulaire. Pour deux d'entre eux, l'extérieur est recouvert d'un engobe blanc. Ils appartiennent tous au même type à panse globulaire et col cylindrique caractéristique des productions provençales ou italiques (fig. 11, no 11 ).

\section{LE MOBILIER MÉTALLIQUE}

\section{Les fibules}

L'ensemble se compose de deux fibules en fer et cinq en bronze. La première est une fibule filiforme en fer de schéma La Tène II à ressort nu de quatre spires et corde interne (fig. 12, $\mathrm{n}^{\circ} 1$ ). L'arc est de forme rectangulaire ininterrompue. Le pied revient sur l'arc, au sommet duquel il se fixe par un étrier ou une bague, sa forme est déjà pratiquement trapézoïdale.

Les deux suivantes constituent des variantes du type Nauheim. L'une est en bronze, le ressort est nu à quatre spires et corde interne (fig. 12, no 3 ). L'arc tendu, de forme trapézoïdale ininterrompue et de section rectangulaire, s'orne d'une profonde rainure médiane et porte en son centre un carré sans doute estampé. Le pied dans le prolongement de l'arc constitue un porte-ardillon de forme trapézoïdale. L'autre, en fer, est incomplète (fig. 12, $\mathrm{n}^{\circ} 2$ ).

Trois autres sont des fibules filiformes en bronze de type La Tène III à ressort nu de quatre spires, et

20 P. Arcelin, Les céramiques de type celtique en Provence, Revue Archéologique de l'Est et du Centre-Est, 32, 1981 , p. 33-66. 

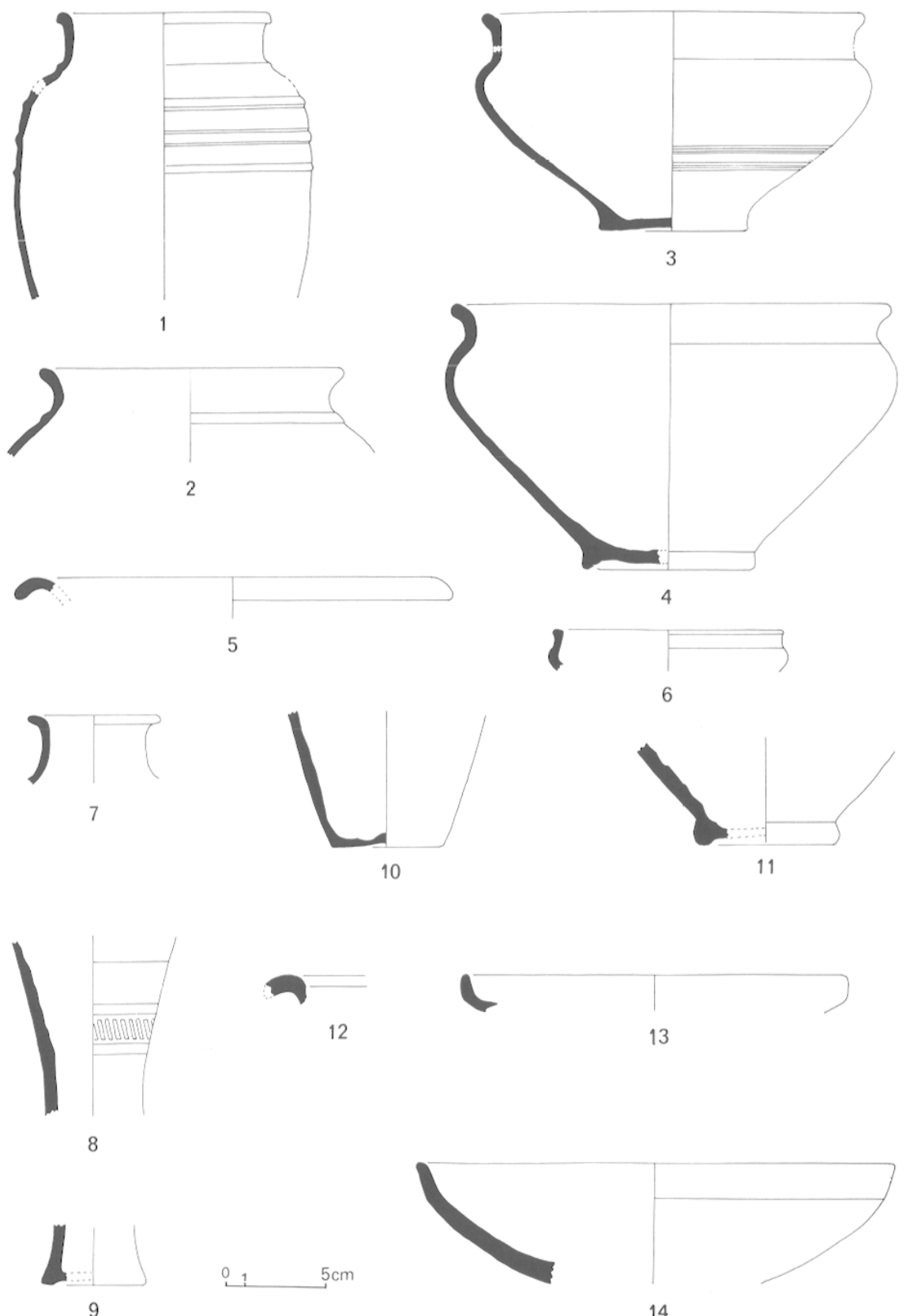

14

Fig. 11 - Céramiques fines. 1-6, céramiques fines lissées; 7-10, céramiques peintes; 11 , céramique à pâte claire; $12-14$, céramiques campaniennes.

corde interne (fig. 12, $\mathrm{n}^{\text {os }} 4$ à 6 ). L'arc est de forme rectangulaire ininterrompue et de section circulaire. Le pied forme un porte-ardillon trapézoïdal. La dernière est trop fragmentaire pour être identifiée (fig. 12, no 7).

\section{Les monnaies}

Le lot comprend quatre potins et deux bronzes frappés : un potin correspond au type BN 5368 attribué aux Séquanes (fig. 13, $\mathrm{n}^{\circ} 1$ ); deux autres appartiennent au type BN 9078 attribué aux Leuques (fig. 13, no 2); le quatrième est illisible.

L'identification de l'un des bronzes avec le type BN 3900, attribué aux Arvernes ne fait aucun doute bien que la légende " $\mathrm{EPAD}$ " caractéristique ne soit pas visible ici (fig. $13, n^{\circ} 3$ ). L'autre bronze est inédit (fig. $13, n^{\circ} 4$ ). Il montre à l'avers une tête à gauche dont les boucles sont stylisées. Au revers, un 

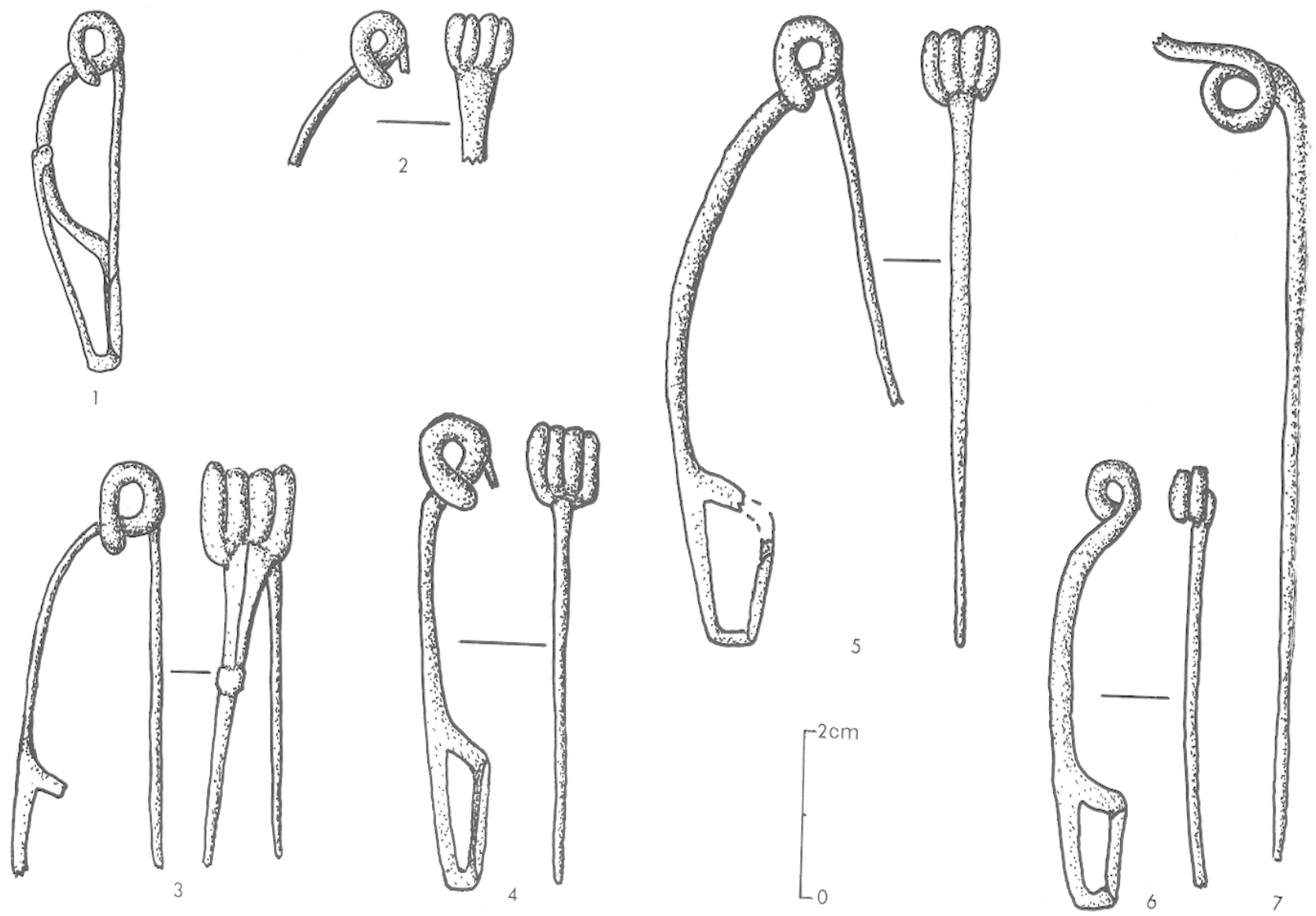

Fig. 12 - Mobilier métallique. 1, 2, fibules en fer; 3-7, fibules en bronze.
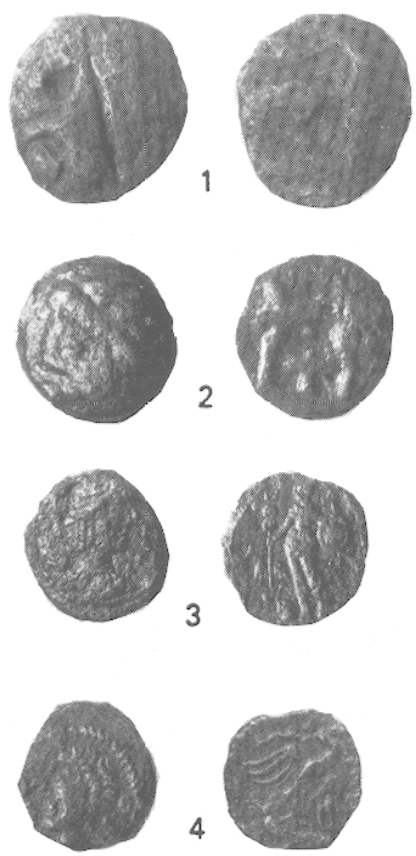

Fig. 13 - Monnaies. 1, potin séquane: 2, potin leuque; 3 , bronze arverne; 4 , bronze inédit (Carnute?). aigle debout ailes déployées surmonte un oiseau. D'après J.-B. Colbert de Beaulieu, il appartiendrait à une des séries de bronzes carnutes.

\section{Le mobilier en fer}

Il se compose de trente fragments de clous à tête plate et section carrée d'environ $10 \mathrm{~cm}$ de longueur, de deux fiches de charpente à tête en équerre et d'un fragment qui pourrait se rapporter à une tarière à bois.

\section{Divers}

Le fossé a également livré deux balles de fronde (fig. $14, \mathrm{n}^{0} 1$ ) et une fusaïole en terre cuite (fig. 14, $n^{\circ} 2$ ), un petit anneau en argent (fig. 14, $n^{\circ} 4$ ) et un fragment d'anneau en bronze (fig. $14, \mathrm{n}^{\circ} 3$ ), un fragment d'épingle (fig. 14, $\mathrm{n}^{\circ} 6$ ), deux perles, l'une en os (fig. $14, n^{0} 5$ ) et l'autre en verre (fig. $\left.14, \mathrm{n}^{0} 7\right)^{21}$.

21 On trouve également un fragment de paroi de verre et une pendeloque en os. Découverts dans un segment perturbé par de nombreuses fosses postérieures, ils constituent sans doute des contaminations. 

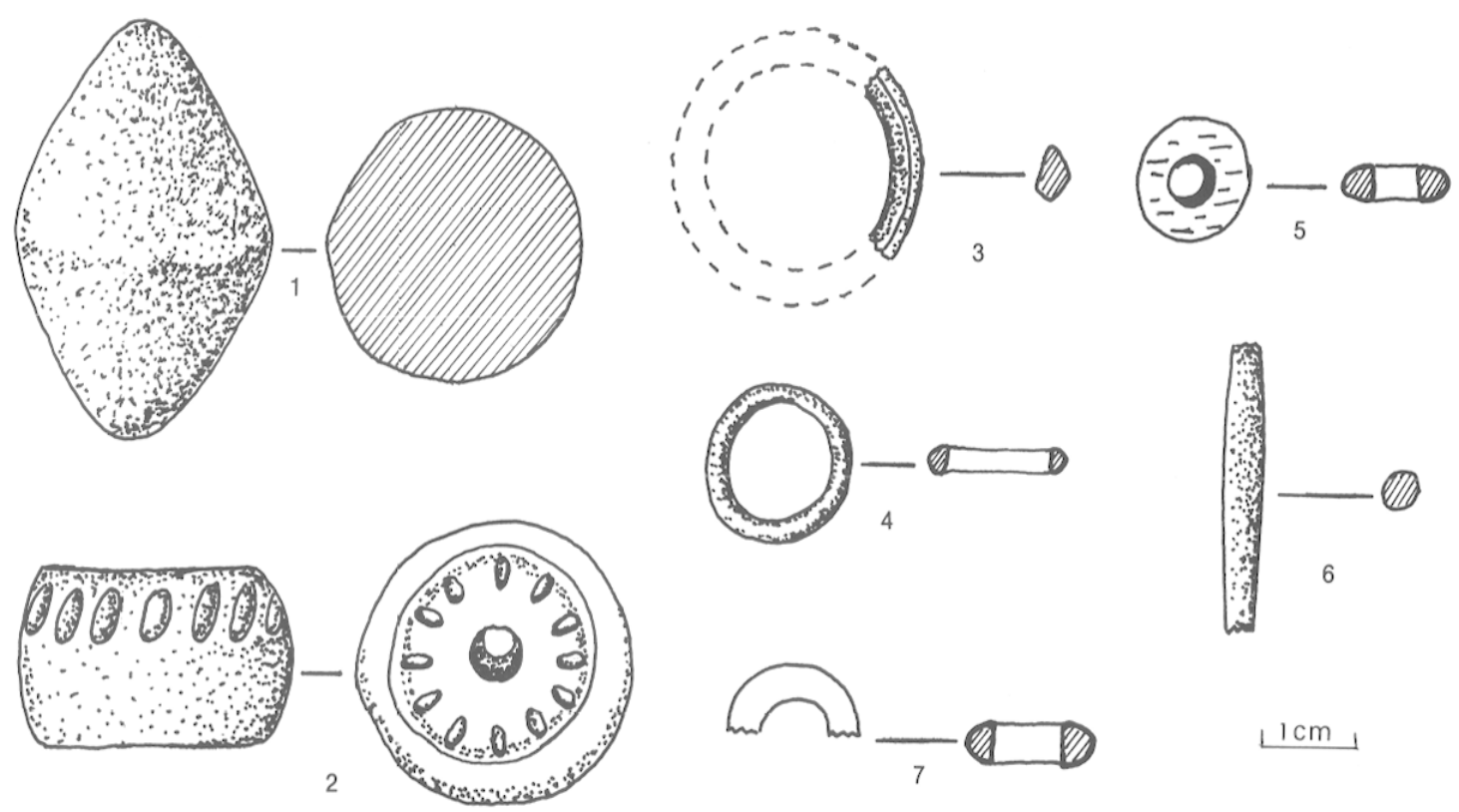

Fig. 14 - Mobilier divers. 1, 2, objets en terre cuite; 3, 4, anneaux métalliques; 5, 6, objets en os; 7 , perle en verre.

\section{Datation du matériel}

La première remarque qui s impose concerne la configuration générale du mobilier, qui, excepté la sur-représentation des amphores, reste conforme à celle de n'importe quel habitat de la même époque. Bien que peu nombreuses, les céramiques indigènes prennent une importance toute particulière du fait de leur affinité très étroite avec les productions de La Tène finale de la Loire (Roanne, Feurs, Goincet).

Les études les plus récentes entreprises sur ces sites ont montré la valeur de la céramique indigène tournée en tant qu'indicateur chronologique; elles ont également mis en lumière le peu de fiabilité des critères de datation traditionnellement admis (amphores, campanienne). Ceux-ci n'apportent en effet, ici que peu de renseignements : l'étude des amphores se heurte à un problème d'ordre typologique et la céramique campanienne n'est pas suffisamment abondante. En revanche, la céramique fine est représentée par des formes bien connues (jattes à profil en $\mathrm{S}$, coupes imitant la forme Lamb. 36 en céramique campanienne, formes hautes en céramique peinte). Ces trois types de vases se retrouvent dans la première période (75-50 av. J.-C.) définie par M. Bessou sur le site de Roanne ${ }^{22}$ et dans la

22 M. Bessou, Étude des vestiges de La Tène découverts à Roanne, fouilles de l'Institution Saint-Joseph, 1976, p. 71. période 3 (100-50 av. J.-C.) établie par M. Vaginay et V. Guichard sur le site de Feurs ${ }^{23}$.

Le lot de fibules, se compose de trois types caractéristiques de La Tène finale : fibule filiforme, fibule de Nauheim et fibule de schéma La Tène moyenne. L'association de ces trois types correspond à la première moitié du $\mathrm{I}^{\text {rr }} \mathrm{s}$. av. J.-C. (cf. Feurs ${ }^{23}$, Bâle ${ }^{24}$ ) et précède directement l'apparition en grand nombre de nouvelles fibules qui sont ici absentes (fibules de type "Stradonice», fibules à coquille et "Kragenfibeln").

En ce qui concerne les monnaies, la présence de deux potins leuques et d'un potin séquane du type A.l défini par A. Furger ${ }^{25}$ renforcent l'analogie déjà constatée avec l'horizon de l'«Usine à gaz» de Bâle ${ }^{24}$. La monnaie attribuée aux Arvernes est, selon G. Gentric, imitée d'un denier romain frappé en 67 av. J.-C. ${ }^{26}$, ce qui fournit un terminus post quem de première importance.

23 M. Vagivay et V. Guichard, L'habilat gaulois de Feurs (Loire), fouilles récentes (1979-1981), Documents d'Archéologie Française, à paraître.

24 A. Furger-Gunti et L. Berger, Katalog und Tafeln der Funde aus der spälkeltischen Siedlung Basel-Gasfabrik, $1980 ;-$ ID., Les sites de l'«usine à gaz» et de la "colline de la cathédrale" à Bâle, L'Age du fer en Europe tempérée, Colloque de Châteauroux, 1978, p. 173-186.

25 A. Furger-Gunti et H. M. von Kafnel, Die Keltische Fundmünzen aus Basel, Schweizerische Numismatische Rundschau, 55, 1976, p. 35-76.

26 G. Gentric et J. Puncet, Monnaies gauloises de Roanne, Cahiers Archéologiques de la Loire, no 45, 1984-85, p. 19. 
L'ensemble du mobilier étudié se rapporte donc globalement aux équivalents régionaux du faciès La Tène D.1 (env. 100-50 av. J.-C.). Mais le terminus indiqué par la monnaie arverne le situe plus vraisemblablement vers la fin dc cettc période.

\section{INTERPRETATION}

Trois séries de faits importants se dégagent de l'ensemble des données exposées ci-dessus :

- Les structures consistent en un réseau de fossés dont le fond était vraisemblablement armé de bois de défense et qui, installés dans la pente bordant le plateau de La Sarra, appartiennent de façon évidente à un ensemble beaucoup plus vaste dont la topographie nous est pour l'instant inconnue. Ce réseau est resté en usage un temps relativement bref. Il ne presente en effet aucune trace de colmatage naturel que le simple cycle des saisons rend inévitable dans un terrain aussi meuble. Nous ne pouvons opposer à cet argument l'hypothèse d'un curage régulier des fossés: cet entretien peu compatible avec la présence des bois de défense, aurait nécessairement laissé des traces. Ces constatations suggèrent une durée d'utilisation relativement brève et dans tous les cas inférieure à un an. Les fossés ont ensuite été comblés volontairement, après, semble-t-il, arrachage des pieux, opération qui ne peut s'interpréter que par le souci de démanteler le dispositif défensif.

- Le mobilier retrouvé dans le fossé aval est constitué pour l'essentiel de résidus de consommation alimentaire utilisés comme matériau de comblement. La conservation des connexions anatomiques constatée sur les restes osseux et l'homogénéité du lot d'amphores témoignent d'un enchaînement rapide dans les opérations de consommation, de collecte, de dépôt et d'enfouissement. D'autres indices, tels que la faible dispersion des tessons ou leur position stratigraphique, indiquent en outre que ces objets ont été collectés directement sur leur lieu de consommation, situé probablement à peu de distance, et qu'ils ont été jetés depuis le bord aval du fossé. Ce dépôt ne peut pas renseigner sur la durée de l'occupation elle-même du fait de la rapidité avec laquelle il s'est constitué. En fait, cette accumulation est déjà la manifestation d'une volonté déclarée de quitter le site car le rejet systématique des détritus dans le fossé constitue un début de colmatage volontaire, contradictoire avec son rôle défensif.
- La composition et le mode de consommation des produits alimentaires reflètent indiscutablement les habitudes indigènes de l'époque mais d'une façon quelque peu particulière comparativement à ce qui est constatć habitucllement sur lcs sites de La Tènc finale. Les amphores, sabrées pour en consommer le vin, et les porcs, débités en quartiers avant d'être rôtis directement sur le feu, représentent une importante consommation d'aliments et de boissons dans un temps très court. Ces faits semblent refléter les pratiques d'une armée en campagne plutôt que relever des mœurs domestiques.

Si le vin ne semble pas faire partie à l'époque républicaine de la ration distribuée aux troupes romaines où il est remplacé par la posca ${ }^{27}$, textes et fouilles montrent à l'évidence que sa consommation n'était pas pour autant proscrite de l'armée, sauf peut-être pendant les expéditions. De même, si l'absence de trace de consommation d'huile fait difficulté, elle n'est pas non plus un argument pour réfuter l'hypothèse du camp romain car le problème se pose dans tous les camps d'époque républicaine fouillés.

La pauvreté du mobilier proprement militaire qui se résume à deux balles de fronde en terre cuite serait un meilleur argument. Quant à la variété des vins consommés, dont quelques-uns pourraient correspondre à des crus réputés mais dont la majorité s'apparente aux vins assez ordinaires d'Étrurie, elle évoque aussi bien les habitudes indigènes que celles des militaires, et ne permet donc pas de trancher ${ }^{28}$.

Il reste que l'arrachage des bois de défense avant comblement des fossés parait bien appartenir aux usages d'une troupe en campagne. De plus, l'exécution des ouvrages défensifs montre une parfaite maitrise des techniques romaines. L'armée n'en a cependant pas l'apanage; cette maitrise dénote un acquis culturel qui peut être le fait de n'importe quel groupe de vétérans ou de troupes ayant servi comme auxiliaire.

Au terme de cette analyse, la seule évidence qui ressort des multiples constatations faites concerne l'aspect défensif et temporaire de cet établissement.

27 Il s'agit d'un mélange de vinaigre et d'eau, cf. Realencyclopädie, XXII-1, p. 420. et A. TchernIA, op. cit, p. 11 à 13 ; sur les problèmes plus généraux du vin et de l'armée, voir A. TChernia, op. cil., p. 13 à 19.

28 Daremberg et Saglio, Dictionnaire des Antiquités, V, Paris, p. 916 ; - A. Tснккnia, Italian wine in Gaul at the end of the Republic, Trade in the Ancient Economy, 1984, p. 92-93; ID., Le vin ..., op. cit, p. 87-90 et 108-109. 
Il reste à en définir la nature. De ce point de vue, l'hypothèse de fossés périphériques à un habitat ou à un sanctuaire ne peut être retenue en raison $d u$ caractère provisoire de cet ouvrage et de la nature même de l'occupation dont témoigne le mobilier. Plus consistante serait l'hypothèse d'un de ces grands banquets rituels rapportés par certains textes $^{29}$; elle cadre cependant assez mal avec l'ampleur de l'ouvrage, son caractère défensif et son démantèlement volontaire. Il ne saurait davantage s'agir de fossés défendant un oppidum car, outre son caractère permanent, ce type d'installation possède une taille et une morphologie bien différentes.

Les données s'accordent en revanche fort bien avec l'hypothèse d'un camp militaire provisoire. Ce type de retranchement, avec les dimensions et les profils caractéristiques de ses fossés, se retrouve sur de nombreux sites militaires : camp républicain de Numance en Espagne, camps augustéens du Limes en Allemagne, camps claudiens en Angleterre, camps césariens d'Alésia et de Nointel et camp augustotibérien d'Aulnay-de-Saintonge en France.

Les rapprochements les plus intéressants peuvent être faits avec le site de Nointel, fouillé et publié par G. Matherat ${ }^{30}$ qui rapporte ces aménagements à la deuxième campagne de César contre les Bellovaques, en 51 av. J.-C. Le site présente un ensemble de camps établis sur un plateau que protège un réseau complexe de retranchements divers aménagés dans les pentes. Nous retrouvons à Nointel les mêmes profils de fossés et les mêmes systèmes de bois de défense qu'au Verbe Incarné. Ces analogies laissent penser que les fossés lyonnais seraient des lignes de défense avancée ou des bracchiae protégeant un camp qu'il reste à découvrir mais qui a toute chance de se trouver sur le plateau de La Sarra.

La mise en œuvre défensive que suggère cette interprétation dépasse à notre avis, celle d'un simple camp de marche. Mais pouvons-nous aller plus loin dans l'identification de ce camp qui a duré, nous l'avons montré, moins d'une année?

Une évaluation des surfaces permet une première approche du problème : la surface nécessaire au campement d'une légion est estimée généralement aux environs de $11 \mathrm{ha}^{31}$. Une telle étendue peut

29 ID., p. 94.

30 G. Matherat, Les techniques des retranchements de César d'après l'enseignement des fouilles de Nointel, Gallia, I, 1943, p. 81-127.

31 ID., p. 101. surprendre, comparée aux surfaces des casernements antiques permanents. Les études anglaises ont montré de façon indiscutable la nette différence entre forts permanents et camps temporaires : les premiers couvrent au plus 5 ha alors que les plus petits des camps repérés en Écosse avoisinent 13 ha et atteignent le plus souvent soit 25 soit $44 \mathrm{ha}^{32}$.

L'ensemble du plateau de La Sarra et de l'éperon de Fourvière offre une superficie comprise entre 24 et 28 ha. Il est donc évident que la totalité des troupes césariennes (au nombre de 5 légions) n'a pas pu cantonner sur ce plateau en 58 av. J.-C. contrairement à l'opinion émise voici quelques lustres par A. Audin ${ }^{33}$. Dans l'hypothèse où César se serait effectivement arrêté à Lyon, ce qui reste à établir, il faudrait imaginer une dispersion des cantonnements sur l'ensemble des hauteurs environnantes. D'autre part, les installations découvertes, quelle que soit l'interprétation proposée (ouvrage avancé, ouvrage de liaison, voire enceinte proprement dite), impliquent, nous l'avons vu précédemment, des aménagements d'une ampleur sans commune mesure avec les besoins d'un avant-poste ou d'une troupe d'éclaireurs en incursion.

\section{$\stackrel{*}{* *}$}

En fait, se posent les problèmes plus généraux de la représentativité de ces quelques mètres de fossé par rapport à un établissement qui couvrait plusieurs hectares, et de la fiabilité d'une datation fondée sur une soixantaine d'objets. A ce propos, il faut évoquer la récente découverte d'un nouveau segment de fossé à l'occasion d'une opération de sauvetage sur le sommet du plateau de La Sarra ${ }^{34}$. Une douzaine de tessons recueillis à cette occasion appartiennent à des productions légèrement postérieures à celles mises en évidence dans le fossé aval; ils conduisent donc à proposer une datation plus récente pour ce segment à savoir aux alentours de 50-30 av. J.-C.

32 D. R. WiLson, Forts et camps romains vus du ciel, extrait des actes du symposium international du C.I.R.A. Bruxelles, 1979, Les Dossiers d'Histoire et d'Archéologie, no 86, 1984, p. 66-70.

33 A. Audin, César et Plancus à Lyon, Album du Crocodile, 1951 ; - ID., Lyon, Miroir de Rome dans les Gaules, Lyon, 1965, p. 29.

34 Nous remercions $M$. Monin, responsable de cette opération menée par le Service Archéologique Municipal de nous avoir fourni ces indications. 
Nous nous acheminons ainsi vers la date de 4443, seule à correspondre à un événement historique attesté, c'est-à-dire la fondation de la ville. Le paysage qu'offrait le plateau à l'époque du creusement des fossés était constitué de prairies hien plus que de forêts ${ }^{35}$. Il a pu inciter aussi bien les colons chassés de Vienne que les fondateurs eux-mêmes à s'installer ici en attendant l'établissement de la nouvelle colonie ${ }^{36}$.

Nous nous garderons pourtant de trancher définitivement dans ce sens pour deux raisons:

- la stratigraphie fait apparaître une phase de désertion entre le comblement des fossés et la première occupation urbaine. La chronologie de

35 Du fait de la nature du terrain et de son acidité, aucun des échantillons prélevés aux fins d'analyses palynologiques n'a livré de grains de pollen. En revanche, les tests de mollusques provenant du lœss aussi bien que ceux contemporains du remblai reflètent un milieu steppique qui affirme le caractère très découvert du plateau à l'époque.

36 Rappelons que, d'après Dion Cassius, le Sénat a ordonné à Lépide et Plancus de fonder la colonie pour établir ceux qui avaient été jadis chassés de Vienne.

N.B. - La photo $\mathrm{n}^{\circ} 3$ est l'œuvre de F. Leyge. l'ensemble est directement subordonnée à la durée et à la datation absolue qu'on assigne à cette phase d'abandon. Mais aucun critère objectif ne permet d'apprécier sa durée. En outre, vouloir lui attribuer une date, suppose qu'on enferme les faciès de céramiques qui la bornent dans des cadres chronologiques d'une précision totalement illusoire;

- en l'absence de liaisons stratigraphiques, la contemporanéité des divers fossés reste sujette à discussion. Si on admet en effet que le site offrait suffisamment d'avantages pour inciter un stratège à s'y installer, il présentait évidemment les mêmes attraits pour ses successeurs; on ne peut donc rejeter l'hypothèse d'une imbrication de camps d'époques différentes, dont la logique nous échappe pour l'instant.

Bernard MandY

avec la collaboration de

Martine Genin

Catherine Godard

Sophie Krausz

Gérard SANDOZ

Philippe ThiRıon 